\title{
Gestational Weight Gain and Risk of Gestational Diabetes Mellitus
}

\author{
Monique M. Hedderson, PhD, Erica P. Gunderson, PhD, and Assiamira Ferrara, MD, PhD \\ Division of Research, Kaiser Permanente Medical Care Program of Northern California, Oakland, \\ California
}

\begin{abstract}
OBJECTIVE-To estimate the relationship between the rate of gestational weight gain before the 50-g, 1-hour oral glucose challenge test screening for gestational diabetes mellitus (GDM) and subsequent risk of GDM.

METHODS-We conducted a nested case-control study (345 women with GDM and 800 women in the control group) within a multiethnic cohort of women delivering between 1996 and 1998 who were screened for GDM at 24-28 weeks of gestation. GDM was diagnosed according to the National Diabetes Data Group plasma glucose cut-offs for the 100-g, 3-hour oral glucose tolerance test. Women's plasma glucose levels, weights, and covariate data were obtained by medical record chart review.
\end{abstract}

RESULTS-After adjusting for age at delivery, race/ethnicity, parity, and prepregnancy body mass index, the risk of GDM increased with increasing rates of gestational weight gain. Compared with the lowest tertile of rate of gestational weight gain (less than $0.27 \mathrm{~kg} / \mathrm{week}$ [less than $0.60 \mathrm{lb} /$ wk]), a rate of weight gain from $0.27-0.40 \mathrm{~kg} / \mathrm{wk}(0.60-0.88 \mathrm{lb} / \mathrm{wk})$ and $0.41 \mathrm{~kg} / \mathrm{wk}(0.89 \mathrm{lb} / \mathrm{wk})$ or more, were associated with increased risks of GDM (odds ratio 1.43, 95\% confidence interval $0.96-2.14$; and odds ratio $1.74,95 \%$ confidence interval 1.16-2.60, respectively). The association between the rate of gestational weight gain and GDM was primarily attributed to increased weight gain in the first trimester. The association was stronger in overweight or obese and nonwhite women.

CONCLUSION—High rates of gestational weight gain, especially early in pregnancy, may increase a woman's risk of GDM. Gestational weight gain during early pregnancy may represent a modifiable risk factor for GDM and needs more attention from health care providers.

\section{LEVEL OF EVIDENCE-II}

Gestational diabetes mellitus (GDM), defined as glucose intolerance with onset or first recognition during the second or third trimester of pregnancy, ${ }^{1}$ complicates $4-7 \%$ of pregnancies in the United States. ${ }^{1}$ Gestational diabetes mellitus is associated with an increased risk of perinatal complications, ${ }^{2}$ and in the long term, women with a history of $\mathrm{GDM}^{3}$ and their offspring ${ }^{4}$ are at high risk of developing type 2 diabetes mellitus.

Weight gain during pregnancy consists of $30 \%$ maternal fat accretion, ${ }^{5}$ with the second half of a normal pregnancy characterized by progressive insulin resistance. ${ }^{6}$ The composition of

\footnotetext{
(C) 2010 by The American College of Obstetricians and Gynecologists. Published by Lippincott Williams \& Wilkins. Corresponding author: Monique Hedderson, PhD, Division of Research, Kaiser Permanente Medical Group, 2000 Broadway, Oakland, CA 94612-2304; mmh@dor.kaiser.org.

Financial Disclosure

The authors did not report any potential conflicts of interest.
} 
weight gain also varies by trimester ${ }^{7,8}$ : maternal weight gain early in pregnancy is disproportionately fat and could influence subsequent maternal insulin resistance. ${ }^{7}$ Surprisingly, few studies have examined the association between gestational weight gain and the risk of GDM. The association must be examined before the diagnosis of GDM to prevent confounding by treatment for GDM. To date, only three studies, with small numbers of women, have examined gestational weight gain from before pregnancy to the glucose screening test for GDM and the risk of abnormal glucose metabolism, with conflicting results. ${ }^{9-11}$

In 2009, the Institute of Medicine (IOM) released new recommendations for gestational weight gain, including specific recommendations for rate of weight gain by prepregnancy body mass index (BMI) (weight in kilograms divided by height in meters squared). ${ }^{12}$ The IOM report noted there was a lack of evidence regarding the role of gestational weight gain in relation to GDM. ${ }^{12}$ It is not known whether weight gains that adhere to these new recommendations can reduce the risk of GDM.

To address this question, we conducted a nested case-control study to examine the occurrence of GDM in relation to the rate of gestational weight gain (in kilograms per week) assessed before the 50-g, 1-hour glucose challenge screening test (referred to as the glucose screening test hereafter) for GDM. We specifically examined the following measures: 1) the overall rate of gestational weight gain, 2) trimester-specific rates of weight gain, and 3) trimester-specific rates of weight gain exceeding compared with adhering to the 2009 IOM recommendations.

\section{MATERIALS AND METHODS}

The study setting is Kaiser Permanente of Northern California, which provides comprehensive medical services to more than 3 million members located in a 14-county region in Northern California. The methods used to identify this cohort have been described in detail elsewhere. ${ }^{13,14}$ Briefly, we identified all pregnancies resulting in a singleton live birth between January 1, 1996, and June 30, 1998, to women without recognized diabetes before pregnancy by using the Kaiser Permanente of Northern California pregnancy glucose tolerance registry, ${ }^{15}$ which has been reported to be $99.4 \%$ accurate. ${ }^{16}$ To be eligible, women could not have had GDM in a previous pregnancy and had to be screened for GDM between 24 and 28 weeks of gestation (according to the earliest ultrasonogram obtained) in the index pregnancy. Trained medical record abstractors completed chart review on the randomly selected women in the case and control groups to confirm the presence of the inclusion criteria. Chart review was conducted on 437 GDM cases identified in the electronic databases, with 388 eligible for study inclusion. We also reviewed the medical records for 1,000 potential women for the control group, of whom 972 met the eligibility criteria. Women were classified as having GDM if two or more plasma glucose values obtained during the 100-g, 3-hour oral glucose tolerance test were abnormal according to the National Diabetes Data Group criteria. ${ }^{17}$

The trained medical record abstracters recorded the pregnancy weights noted on the prenatal form, which included self-reported prepregnancy weight, any measured weights during the 1 year before pregnancy, weight measured at the first prenatal visit, and weight measured at or before the patient had her 50-g, 1-hour glucose challenge screening test for GDM. Additional information obtained from the medical records included height, last menstrual period (LMP), parity, smoking during pregnancy, blood pressure at the first prenatal visit, and gestational age estimated by the earliest ultrasonogram obtained before 24 weeks of gestation. Blood pressure at the first prenatal visit was categorized according to the American Heart Association's criteria. ${ }^{18}$ Preeclampsia was considered present if a woman 
had a physician's diagnosis in the medical chart. Women's self-reported race/ethnicity and education were obtained from the neonate's birth certificate.

The rate of gestational weight gain, per week, before the glucose screening test was calculated as the weight measured at or before the glucose screening test minus prepregnancy weight divided by the weeks of gestation attained at the time of the weight measurement. On average, the glucose screening test weight was measured less than 1 week before the test (mean [ \pm standard deviation (SD)]0.8 $[ \pm 1.1]$ weeks, range $0-4$ weeks). We calculated the rate of gestational weight gain in the first trimester as the weight at first prenatal visit (12 $[ \pm 1.5]$ weeks, range $6-13$ weeks) minus self-reported pregravid weight divided by the weeks of gestation attained at the first prenatal visit. Rate of weight gain in the second trimester was defined as the weight at glucose screening test (performed on average at $26[ \pm 1.8]$ weeks) minus weight at the first prenatal visit divided by the number of weeks between measurements. The gestational week assigned to each maternal weight measurement was based on the earliest ultrasonogram obtained before 24 weeks of gestation.

The 2009 IOM recommendations provide pre-gravid BMI-specific recommendations for a range of absolute weight gain in the first trimester and a range of rate of weight gain per week for the second and third trimesters. ${ }^{12}$ Based on the observed weight gain at the time of the 1-hour glucose challenge test, we determined whether each woman met, exceeded, or was below the IOM recommendations. We subtracted 13 weeks from the gestational age at the time of the glucose screening test and multiplied this value by the BMI-specific recommended rate of weight gain for the second and third trimesters. Weight gained after the first trimester was then added to the BMI-specific absolute weight gain recommended by the IOM for the first trimester.

Self-reported prepregnancy weight was missing for 226 of the eligible women (15\%). For those missing a self-reported prepregnancy weight, the measured weight closest to the woman's LMP but no more than 12 months before her LMP, was used. To validate this method of estimating prepregnancy weight, we compared the self-reported prepregnancy weight to a weight measured within 12 months of the LMP among 507 women $(44.7 \%)$ for whom both measurements were available. The intraclass correlation coefficient between the two weights was 0.967 . The mean difference (self-reported minus measured weight) was 1.2 $( \pm 3.5) \mathrm{kg}$ for normal weight women, $0.8( \pm 4.9) \mathrm{kg}$ for overweight women, and $-1.3( \pm 5.0)$ $\mathrm{kg}$ for obese women. These findings are similar to previous reports, ${ }^{19-21}$ except that unlike previous studies, ${ }^{19,21}$ the obese women in our study were less likely to under-report their pregravid weight.

Of the eligible 388 women with GDM and 972 women in the control group for whom chart review was completed, the following were excluded from all analyses because of missing data: pregravid weight (10.6\% of women in the case group and $15.4 \%$ of women in the control group), height ( $0 \%$ of women in the case group and $0.7 \%$ of women in the control group), and no weight measured at a prenatal visit within 4 weeks of screening test (1.7\% of women in the case group and $2.8 \%$ of women in the control group). This left 341 women with GDM and 793 women in the control group for analyses. Women for whom gestational weight gain data were missing were similar to those included in the analysis regarding age, race/ethnicity, parity, and education. This study was approved by the human subjects committee of the Kaiser Foundation Research Institute and the Committee for the Protection of Human Subjects, California Health and Human Services Agency.

We categorized women into tertiles based on the distribution of the rate of weight gain in the women in the control group. We examined differences between women in the case group 
and women in the control group in the distributions of categorical variables using $\chi^{2}$ tests. Unconditional logistic regression was used to obtain odds ratios (ORs) as estimates of the relative risk of GDM in relation to category of rate of gestational weight gain. Women in the lowest third of the distribution of rate of gestational weight gain were used as the reference group. For models using the IOM recommendations as the exposure, we compared women who exceeded the recommendations with those who met or were below the recommendations because so few women were below the recommendations (46 women in the case group [13\%] and 104 women in the control group [13\%]). Variables evaluated for confounding included maternal age, race/ethnicity, pregravid BMI, blood pressure at the first prenatal visit, parity, maternal education, and first-degree family history of diabetes. To assess confounding, we entered covariates into a logistic regression model one at a time and then compared the adjusted and unadjusted odds ratios. ${ }^{22}$ Final logistic regression models included covariates that altered unadjusted odds ratios for gestational weight gain by at least $10 \%$, as well as those covariates of a priori interest (ie, parity and blood pressure). The multivariable adjusted models included the following covariates: age, race/ethnicity, parity, blood pressure, and prepregnancy BMI. Sensitivity analyses were conducted to examine first-trimester weight gain and weight gain up to the time of glucose screening among those women with a measured weight that was within 6 months of their LMP and further adjusted for the length of time between the prepregnancy weight measurement and the index pregnancy. In addition, we also conducted a sensitivity analysis examining the rate of gain in the first trimester among women who had their first prenatal visit performed at 12-14 weeks of gestation to assess whether measuring weight at the end of the first trimester would change our results. To assess the potential modifying effects of pregravid BMI (continuous) and race/ethnicity (non-Hispanic white compared with African-American, Asian, and Hispanic women) and age (younger than 30 years compared with 30 years or older), we examined interaction terms and repeated the analyses within these subgroups.

\section{RESULTS}

The characteristics of women with GDM and women in the control group are listed in Table 1. Women with GDM were more likely to be from a nonwhite race/ethnicity group, to be older than 35 years, to have a family history of diabetes, to have hypertension at the first prenatal visit, to have had two or more prior live births, and to be overweight or obese before pregnancy. Women with GDM tended to have a higher occurrence of preeclampsia and to have 12 or fewer years of education, although these differences were likely attributable to chance. Overall, the median rate of gestational weight gain from prepregnancy until the glucose screening test was $0.33 \mathrm{~kg} / \mathrm{wk}(0.73 \mathrm{lb} / \mathrm{wk})$, the median rate of gestational weight gain until the first prenatal visit (12 weeks of gestation on average) was $0.18 \mathrm{~kg} / \mathrm{wk}(0.40 \mathrm{lb} / \mathrm{wk})$, and the median rate of gestational weight in midpregnancy (from the first prenatal visit until the 1-hour glucose challenge test) was $0.44 \mathrm{~kg} / \mathrm{wk}(0.97 \mathrm{lb} /$ wk). The mean rate of weight gain until the glucose screening test varied by pregravid BMI and by case-control status. The mean $( \pm \mathrm{SD})$ rate of weight gain was highest among women with a BMI of $24.9 \mathrm{~kg} / \mathrm{m}^{2}$ or less: $0.38( \pm 0.14) \mathrm{kg} / \mathrm{wk}(0.84[ \pm 0.31] \mathrm{lb} / \mathrm{wk})$ for women in the case group and $0.37( \pm 0.16) \mathrm{kg} / \mathrm{wk}(0.82[ \pm 0.35] \mathrm{lb} / \mathrm{wk})$ for women in the control group. The mean $( \pm \mathrm{SD})$ rate of weight gain was second highest among overweight women with a BMI of $25.0-29.9 \mathrm{~kg} / \mathrm{m}^{2}: 0.36( \pm 0.20) \mathrm{kg} / \mathrm{wk}(0.79[ \pm 0.44] \mathrm{lb} / \mathrm{wk})$ for women in the case group and $0.31( \pm 0.17) \mathrm{kg} / \mathrm{wk}(0.68[ \pm 0.37] \mathrm{lb} / \mathrm{wk})$ for women in the control group. The mean $( \pm \mathrm{SD})$ rate of weight gain was lowest among obese women with a BMI of $30 \mathrm{~kg} / \mathrm{m}^{2}$ or more: $0.26( \pm 0.21) \mathrm{kg} / \mathrm{wk}(0.57[ \pm 0.46] \mathrm{lb} / \mathrm{wk})$ for women in the case group and 0.21 $( \pm 0.26) \mathrm{kg} / \mathrm{wk}(0.46[ \pm 0.57] \mathrm{lb} / \mathrm{wk})$ for women in the control group.

In the unadjusted models, there was no association between tertile of rate of gestational weight gain up to the glucose screening test and risk of GDM. However, after adjustment for 
BMI, age, race/ethnicity, parity, and blood pressure at the first prenatal visit, women in the middle and highest tertiles for rate of weight gain were more likely to develop GDM when compared with women in the lowest tertile (Table 2). To exclude the possibility that gestational weight gain was a prodromic symptom of preeclampsia, which is also more common in women with GDM, ${ }^{18}$ we reran the analysis after excluding the 19 women in the case group (5.5\%) and 26 women in the control goup (3.3\%) who had developed preeclampsia. The observed associations between gestational weight gain and GDM risk did not change direction and remained statistically significant: ORs for middle and highest tertiles were 1.5 (95\% confidence interval [CI] 1.0-2.1) and 1.7 (95\% CI 1.2-2.5), respectively. We conducted sensitivity analyses restricted to women with a measured weight within 6 months of their LMP (158 GDM women in the case group and 250 women in the control group), further adjusted for the time between the weight measurement and the index pregnancy, and found that the results were only slightly attenuated (OR 1.4 [95\% CI 0.92.1] and OR 1.5 [95\% CI 1.1-2.2] for middle and highest tertiles, respectively).

We also examined the relationship between trimester-specific rates of gain and GDM (Table 2). Being in the highest tertile for rate of weight gain in the first trimester was associated with a significant, $80 \%$ significant increased risk of GDM. The strength of the association increased in a sensitivity analysis restricted to women who had their weight measured within 6 months before their LMP (OR 2.31, 95\% CI 1.32-4.04, for being in the highest tertile). In the sensitivity analysis restricted to those who had their first prenatal visit performed between 12 and 14 weeks of gestation, the risk associated with being in the highest tertile for rate of gestational weight gain in the first trimester increased to twofold (OR $2.18,95 \%$ CI 1.17-4.08). There was no association between the rate of weight gain during the second trimester and GDM. Women who exceeded the IOM guidelines for weight gain had a significant, $50 \%$ increase in the risk of GDM when compared with women who gained within or below the IOM recommendations (Table 2).

We examined interaction terms between several covariates (BMI, age, and race/ethnicity). The interaction term for pregravid BMI and the rate of gestational weight gain up to the time of GDM screening was statistically significant $(P<.05)$. Therefore, we examined the association between the rate of weight gain up to the screening test and risk of GDM within strata of prepregnancy BMI categories. Because of the sparse number of underweight women (BMI less than 18.5), they were combined with the normal-weight women (BMI 19.0-24.9) for this analysis. In multivariable analyses, the association appeared to be stronger among overweight women. Overweight women who gained in the highest tertile for rate of weight gain $(0.41-0.97 \mathrm{~kg} / \mathrm{wk})$ were twice as likely (OR 2.1, 95\% CI 1.05-4.06) to develop GDM when compared with women in the lowest tertile. The corresponding odds ratios for normal weight and obese women were 1.41 (95\% CI 0.77-2.58) and 1.18 (95\% CI 0.57-2.44), respectively (Fig. 1).

The interaction term for race/ethnicity (non-Hispanic white compared with Asian, Hispanic, and African-American women) and the rate of gestational weight gain up to the time of GDM screening attained borderline significance $(P=.05)$. Stratified analyses suggested that the association between the rate of weight gain before the GDM screening and the risk of GDM was stronger among nonwhite women. Women of nonwhite race/ethnicity who were in the highest tertile for rate of weight gain had a 2.5-fold increased risk of developing GDM (OR 2.66, 95\% CI 1.45-4.90), whereas the corresponding value for non-Hispanic white women was OR 1.56 (95\% CI 0.90-2.68). There were no significant interactions between rate of weight gain in the first trimester or rate of weight gain in the second trimester. 


\section{DISCUSSION}

In this study, we found that greater gestational weight gain in early pregnancy, particularly during the first trimester, was associated with an increased risk of GDM. Exceeding the 2009 IOM recommendations for gestational weight gain was also associated with an increased risk of developing GDM. All of these associations were independent of important confounders, including age, race/ethnicity, pregravid BMI, and parity. However, the association between gestational weight gain and the risk of GDM was more pronounced among overweight and nonwhite women.

Strengths of this study include the representative population, the robustness of the results after several sensitivity analyses, and the biologic plausibility of a stronger association between gestational weight gain during the first trimester and the risk of GDM. ${ }^{7}$ However, several limitations should be considered when interpreting the results of this study. First, we used prepregnancy weights that were (for most women) self-reported. Although we and other investigators have observed that self-reported prepregnancy weight approximates the true value, ${ }^{19,23}$ we may have underestimated or overestimated the association with GDM. However, our sensitivity analyses restricted to women with measured weights were encouraging. We have no reason to believe that the women who developed GDM would have underreported their prepregnancy weight more than control women because none of the women included had a history of GDM. Thus, we expect that any bias attributable to the misclassification of prepregnancy BMI would be nondifferential and would therefore bias our results toward the null. We were also missing data on weights recorded in the medical charts for approximately $15 \%$ of the women. If women with missing data were more or less likely to gain excessive weight, we could have underestimated or overestimated the risk of GDM associated with gestational weight gain. We did not have information on the composition of pregnancy weight gain, and it is plausible that gaining fat mass compared with lean mass could have differential effects on GDM risk. Finally, we lacked information on some potential confounders, such as diet and physical activity, so we were not able to assess the association between gestational weight gain and GDM independent of these factors.

Three prior studies have found that excessive gestational weight gain is associated with an increased risk of impaired glucose tolerance during pregnancy that did not meet the strict criteria for GDM. ${ }^{9-11}$ Similar to our finding that the association was stronger among overweight and obese women, one study found that a higher observed-to-expected gestational weight gain ratio increased the risk of impaired glucose tolerance only among overweight women, ${ }^{10}$ and another study found that exceeding the 1990 IOM recommendations increased the risk of impaired glucose tolerance, but only among obese women. ${ }^{9}$ A recent study ${ }^{11}$ found no association between gestational weight gain before the GDM screening and risk of GDM, but they did find a borderline association between a high rate of weight gain in the first trimester and risk of GDM (OR 1.70, 95\% CI 0.98-2.94). The smaller sample size of these previous studies may explain some of the discrepancies, but overall prior studies support our findings. We tested and found an interaction between gestational weight gain and race/ethnicity, suggesting that excessive gestational weight gain may have a greater effect on the risk of GDM in nonwhite women than in white women.

We found that the association between gestational weight gain and risk of GDM was mainly attributable to excessive weight gain in the first trimester. Rapid gestational weight gain in early pregnancy may result in an early increase in insulin resistance that leads to the "exhaustion" of the B cell. This could reduce the capacity of the B cells to secrete adequate levels of insulin to compensate for the insulin resistance induced by the progression of pregnancy and therefore lead to the development of GDM. Although we were unable to 
measure the components of gestational weight gain (eg, maternal fat mass, fat-free mass, mass of the fetus), weight gain in early pregnancy has been found to consist of more maternal body fat. ${ }^{7,8}$ Large gains in maternal fat mass in early pregnancy could have a stronger influence on subsequent insulin resistance and, consequently, GDM risk than later gains in lean tissue or fetal mass. Catalano et $\mathrm{al}^{24}$ found an inverse association between maternal fat accretion and insulin sensitivity from before conception through 12 to 14 weeks of gestation, but not from early to late pregnancy. The authors speculated that at 12 to 14 weeks of gestation they were primarily estimating changes in maternal metabolism, and only a small proportion of the change was attributable to increased fetal or placental tissue.

The 2009 IOM pregnancy weight gain report suggested that more evidence was needed to determine whether gestational weight gain plays a role in the cause of GDM. ${ }^{12}$ Our study is one of the first to support a direct association between gestational weight gain and the risk of GDM. These findings need to be replicated in other racial/ethnically diverse populations. Clinicians should be aware that high rates of weight gain during early pregnancy may increase a woman's risk of GDM, particularly among overweight or obese women.

Avoidance of excessive weight gain early in pregnancy may be an effective strategy for prevention of GDM, but randomized studies are needed to determine the feasibility of such an early intervention and the best methods to help women meet the IOM recommendations for gestational weight gain.

\section{Acknowledgments}

Supported by National Institute of Diabetes and Digestive and Kidney Diseases grant R01 DK 54834 and a grant from the American Diabetes Association to Dr. Ferrara.

\section{References}

1. American Diabetes Association. Gestational diabetes mellitus. Diabetes Care. 2001; 23(suppl 1):S77-9.

2. Metzger BE. Summary and recommendations of the Third International Workshop-Conference on Gestational Diabetes Mellitus. Diabetes. 1991; 40(suppl 2):197-201. [PubMed: 1748259]

3. O'Sullivan JB, Mahan CM. Criteria for the oral glucose tolerance test in pregnancy. Diabetes. 1964; 13:278-85. [PubMed: 14166677]

4. Silverman BL, Rizzo TA, Cho NH, Metzger BE. Long-term effects of the intrauterine environment. The Northwestern University Diabetes in Pregnancy Center. Diabetes Care. 1998; 21(suppl 2):B142-9. [PubMed: 9704242]

5. Institute of Medicine, Subcommittee on Nutritional Status and Weight Gain during Pregnancy. Nutrition during pregnancy. Washington, DC: National Academy of Sciences; 1990.

6. Buchanan TA, Xiang AH. Gestational diabetes mellitus. J Clin Invest. 2005; 115:485-91. [PubMed: 15765129]

7. van Raaij JM, Peek ME, Vermaat-Miedema SH, Schonk CM, Hautvast JG. New equations for estimating body fat mass in pregnancy from body density or total body water. Am J Clin Nutr. 1988; 48:24-9. [PubMed: 3389327]

8. Hytten, FE.; Chamberlain, G. Clinical physiology in obstetrics. Oxford, Boston: Blackwell Scientific Publications; 1980.

9. Tovar A, Must A, Bermudez OI, Hyatt RR, Chasan-Taber L. The impact of gestational weight gain and diet on abnormal glucose tolerance during pregnancy in Hispanic women. Matern Child Health J. 2009; 13:520-30. [PubMed: 18597166]

10. Saldana TM, Siega-Riz AM, Adair LS, Suchindran C. The relationship between pregnancy weight gain and glucose tolerance status among black and white women in central North Carolina. Am J Obstet Gynecol. 2006; 195:1629-35. [PubMed: 16824460] 
11. Herring SJ, Oken E, Rifas-Shiman SL, Rich-Edwards JW, Stuebe AM, Kleinman KP, et al. Weight gain in pregnancy and risk of maternal hyperglycemia. Am J Obstet Gynecol. 2009; 201:61.e1-7. [PubMed: 19371858]

12. Weight gain during pregnancy: Reexamining the guidelines. Washington, DC: National Academy Press; 2009.

13. Hedderson MM, Ferrara A, Williams MA, Holt VL, Weiss NS. Androgenicity of progestins in hormonal contraceptives and the risk of gestational diabetes mellitus. Diabetes Care. 2007; 30:1062-8. [PubMed: 17303784]

14. Hedderson MM, Ferrara A. High blood pressure before and during early pregnancy is associated with an increased risk of gestational diabetes mellitus. Diabetes Care. 2008; 31:2362-7. [PubMed: 18809624]

15. Ferrara A, Kahn HS, Quesenberry C, Riley C, Hedderson MM. An increase in the incidence of gestational diabetes mellitus: Northern California, 1991-2000. Obstet Gynecol. 2004; 103:526-33. [PubMed: 14990417]

16. Ferrara A, Weiss NS, Hedderson MM, Quesenberry CP Jr, Selby JV, Ergas IJ, et al. Pregnancy plasma glucose levels exceeding the American Diabetes Association thresholds, but below the National Diabetes Data Group thresholds for gestational diabetes mellitus, are related to the risk of neonatal macrosomia, hypoglycaemia and hyperbilirubinaemia. Diabetologia. 2007; 50:298-306. [PubMed: 17103140]

17. American Diabetes Association. Gestational diabetes mellitus. Diabetes Care. 1998; 21(suppl 1):S60-1.

18. Lichtenstein AH, Appel LJ, Brands M, Carnethon M, Daniels S, Franch HA, et al. Diet and lifestyle recommendations revision 2006: a scientific statement from the American Heart Association Nutrition Committee. Circulation. 2006; 114:82-96. [PubMed: 16785338]

19. Yu SM, Nagey DA. Validity of self-reported pregravid weight. Ann Epidemiol. 1992; 2:715-21. [PubMed: 1342323]

20. Stevens-Simon C, Roghmann KJ, McAnarney ER. Relationship of self-reported prepregnant weight and weight gain during pregnancy to maternal body habitus and age. J Am Diet Assoc. 1992; 92:85-7. [PubMed: 1728630]

21. Gunderson EP, Abrams B, Selvin S. Does the pattern of postpartum weight change differ according to pregravid body size? Int J Obes Relat Metab Disord. 2001; 25:853-62. [PubMed: 11439300]

22. Rothman, KJ.; Greenland, S. Modern epidemiology. 2. Philadelphia (PA): Lippincott-Raven Publishers; 1998.

23. Harris HE, Ellison GT. Practical approaches for estimating prepregnant body weight. J Nurse Midwifery. 1998; 43:97-101. [PubMed: 9581094]

24. Catalano PM, Roman-Drago NM, Amini SB, Sims EA. Longitudinal changes in body composition and energy balance in lean women with normal and abnormal glucose tolerance during pregnancy. Am J Obstet Gynecol. 1998; 179:156-65. [PubMed: 9704782] 


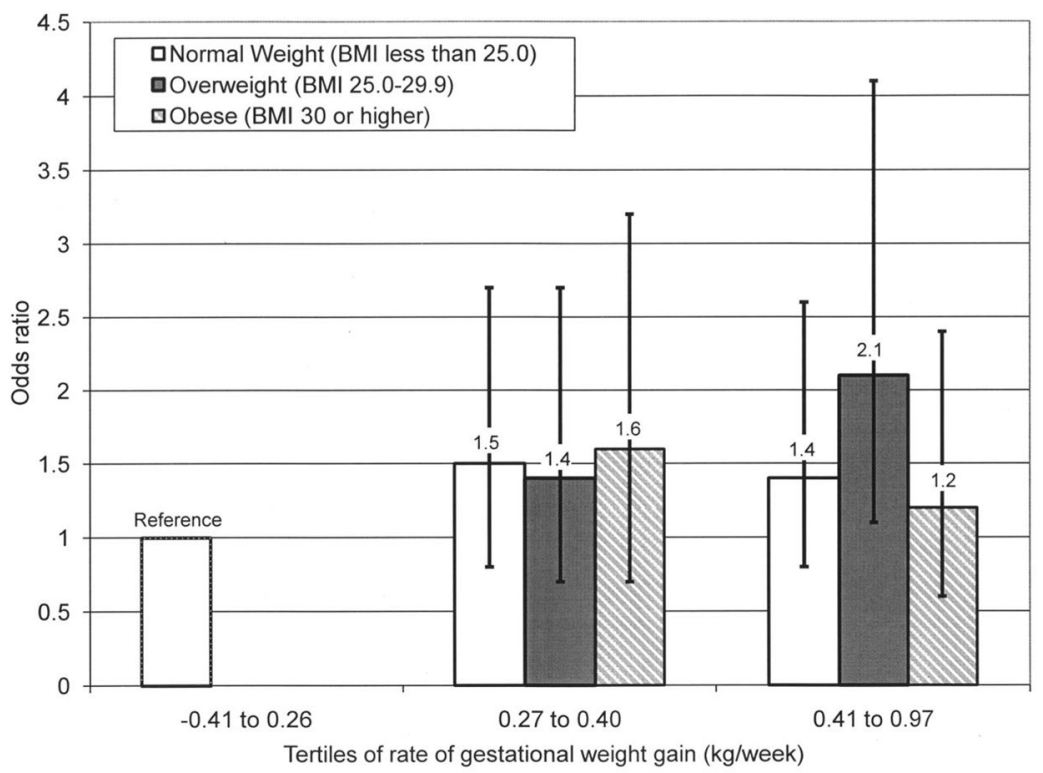

Fig. 1.

Odds ratios and $95 \%$ confidence intervals for gestational diabetes mellitus associated with rate of gestational weight gain up to screening test by body mass index (BMI). Normal weight, BMI less than 25.0; overweight, BMI 25.0-29.9; obese, BMI 30 or more. Hedderson. Gestational Weight Gain and GDM. Obstet Gynecol 2010. 
Table 1

Characteristics of the Study Population: Kaiser Permanente of Northern California, January 1996-June 1998

\begin{tabular}{|c|c|c|c|c|}
\hline Characteristic & Gestational Diabetes Cases* $(n=341)$ & Controls $(n=793)$ & Missing Data $(\mathrm{n}=\mathbf{2 2 6})$ & $P^{*}$ \\
\hline Race/ethnicity & & & & .003 \\
\hline Non-Hispanic white & $153(44.9)$ & $431(54.4)$ & $99(43.8)$ & \\
\hline African American & $27(7.9)$ & $80(10.1)$ & $18(7.0)$ & \\
\hline Asian & $41(12.0)$ & $64(8.1)$ & $28(12.4)$ & \\
\hline Hispanic & $65(19.1)$ & $133(16.8)$ & $50(22.1)$ & \\
\hline Other & $55(16.1)$ & 85 (10.7) & $31(13.7)$ & \\
\hline Age at delivery (y) & & & & $<.001$ \\
\hline $15-24$ & $23(6.7)$ & $189(23.8)$ & $50(22.1)$ & \\
\hline $25-29$ & $48(14.1)$ & $189(23.8)$ & $51(22.5)$ & \\
\hline $30-34$ & $114(33.4)$ & $251(31.7)$ & $30(35.4)$ & \\
\hline $35-45$ & $156(45.8)$ & $164(20.7)$ & $45(19.9)$ & \\
\hline \multicolumn{5}{|l|}{ BMI $\left(\mathrm{kg} / \mathrm{m}^{2}\right)$} \\
\hline Less than 18.5 & $9(2.6)$ & $39(5.0)$ & - & $<.001$ \\
\hline $18.5-24.9$ & $109(32.0)$ & $450(58.1)$ & - & \\
\hline $25.0-29.9$ & $118(34.6)$ & $178(23.0)$ & - & \\
\hline 30.0 or more & $105(30.8)$ & $108(13.9)$ & - & \\
\hline Parity & & & & .02 \\
\hline 0 & $134(39.3)$ & $345(43.5)$ & $87(38.5)$ & \\
\hline 1 & $113(33.1)$ & $288(36.3)$ & $81(35.8)$ & \\
\hline 2 or more & 94 (27.6) & $160(20.2)$ & $58(25.7)$ & \\
\hline Educational attainment & & & & .13 \\
\hline High school graduate or lower & $123(36.1)$ & $310(39.1)$ & $102(45.5)$ & \\
\hline Partial college & $110(32.3)$ & $233(29.4)$ & $71(31.7)$ & \\
\hline College & $72(21.1)$ & $138(17.4)$ & $28(12.5)$ & \\
\hline Graduate school or higher & $31(9.1)$ & $101(12.7)$ & $23(10.3)$ & \\
\hline Do not know & $5(1.5)$ & $11(1.4)$ & $2(0.01)$ & \\
\hline Marital status & & & & $<.001$ \\
\hline Single & $46(13.7)$ & $197(25.0)$ & $45(20)$. & \\
\hline Married & $271(80.4)$ & $572(72.5)$ & $174(77.3)$ & \\
\hline Widowed, divorced, or separated & $14(4.1))$ & $18(2.2)$ & $6(2.7)$ & \\
\hline Do not know & $6(1.8)$ & $2(0.3)$ & $1(0.0)$ & \\
\hline Family history of diabetes & & & & $<.001$ \\
\hline First-degree relative & $79(23.2)$ & $88(11.1)$ & $32(14.2)$ & \\
\hline Second-degree relative & $92(27.0)$ & $180(22.7)$ & $38(14.8)$ & \\
\hline None & $135(39.6)$ & $470(59.3)$ & $127(56.2)$ & \\
\hline Do not know & $35(10.2)$ & $55(6.9)$ & $29(12.8)$ & \\
\hline \multicolumn{5}{|l|}{ Rate of weight gain $\left(\mathrm{kg} / \mathrm{m}^{2}\right)$} \\
\hline Prepregnancy to 1-hour GCT & $0.34(0.19)$ & $0.34(0.18)$ & - & \\
\hline First trimester & $0.25(0.35)$ & $0.17(0.32)$ & - & \\
\hline
\end{tabular}




\begin{tabular}{|c|c|c|c|c|}
\hline Characteristic & Gestational Diabetes Cases* $(n=341)$ & Controls $(n=793)$ & Missing Data (n=226) & $P^{*}$ \\
\hline Second trimester & $0.40(0.21)$ & $0.47(0.22)$ & - & \\
\hline \multicolumn{5}{|l|}{ IOM recommendations } \\
\hline Below & $46(13.5)$ & $104(13.1)$ & - & .21 \\
\hline Met & $50(14.7)$ & $151(19.0)$ & - & \\
\hline Exceed & $245(71.9)$ & $538(67.8)$ & - & \\
\hline Preeclampsia diagnosis & $19(5.6)$ & $26(3.4)$ & $3(1.4)$ & .10 \\
\hline Blood pressure at first prenatal visit & & & & $<.001$ \\
\hline Normal & $175(51.3)$ & $554(70.1)$ & $155(69.5)$ & \\
\hline Prehypertension & $129(37.8)$ & $207(26.2)$ & $59(26.5)$ & \\
\hline Hypertension & $37(10.9)$ & $29(3.7)$ & $9(4.0)$ & \\
\hline
\end{tabular}

BMI, body mass index; IOM, Institute of Medicine; GCT, glucose challenge test.

Data are $\mathrm{n}(\%)$.

* Compares cases and controls using the $\chi^{2}$ test. 
Table 2

Odds Ratios and 95\% Confidence Intervals for Gestational Diabetes Mellitus Associated With Rate of Pregnancy Weight Gain

\begin{tabular}{|c|c|c|c|c|}
\hline & Cases & Control & Crude OR (95\% CI) & Adjusted OR ${ }^{*}(95 \%$ CI $)$ \\
\hline \multicolumn{5}{|c|}{ Rate of weight gain up to GDM screening (kg/wk) } \\
\hline$-0.41-0.26$ (ref) & $114(33.4)$ & $261(32.9)$ & 1.0 & 1.0 \\
\hline $0.27-0.40$ & $112(32.8)$ & $269(33.9)$ & $0.95(0.70-1.30)$ & $1.43(0.96-2.14)$ \\
\hline $0.41-0.97$ & $115(33.7)$ & $263(33.2)$ & $1.00(0.74-1.37)$ & $1.74(1.16-2.60)$ \\
\hline \multicolumn{5}{|c|}{ Rate of weight gain in first trimester (kg/wk) } \\
\hline$-1.0-0.06$ & $100(29.2)$ & $269(32.8)$ & 1.0 & 1.0 \\
\hline $0.07-0.26$ & $99(28.9)$ & 278 (33.9) & $0.96(0.69-1.32)$ & $1.04(0.70-1.56)$ \\
\hline $0.27-1.9$ & $144(42.0)$ & $273(33.3)$ & $1.42(1.05-1.93)$ & $1.82(1.24-2.70)$ \\
\hline \multicolumn{5}{|c|}{ Rate of weight gain in second trimester (kg/wk) } \\
\hline$-1.0-0.38$ & $162(42.9)$ & $295(32.6)$ & 1.0 & 1.0 \\
\hline $0.39-0.54$ & $125(33.1)$ & $307(34.0)$ & $0.74(0.56-0.98)$ & $1.02(0.70-1.49)$ \\
\hline $0.55-1.62$ & $91(24.1)$ & $302(33.4)$ & $0.55(0.41-0.74)$ & $0.88(0.59-1.33)$ \\
\hline \multicolumn{5}{|c|}{ IOM recommendations } \\
\hline Below/within & $96(28.2)$ & $255(32.2)$ & 1.0 & 1.0 \\
\hline Above & 245 (71.9) & $538(67.8)$ & $1.38(1.09-1.77)$ & $1.53(1.09-2.15)$ \\
\hline \multicolumn{5}{|c|}{ OR, odds ratio; CI, confidence interval; GDM, gestational diabetes mellitus; IOM, Institute of Medicin } \\
\hline
\end{tabular}

